\title{
Notification List \\ Notification that new names and new combinations have appeared in volume 55, part 1, of the IJSEM
}

Correspondence

Jean Euzéby

email address can be found at

www.bacterio.net
This listing of names published in a previous issue of the IJSEM is provided as a service to bacteriology to assist in the recognition of new names and new combinations. This procedure was proposed by the Judicial Commission [Minute 11(ii), Int J Syst Bacteriol 41 (1991), p. 185]. The names given herein are listed according to the Rules of priority (i.e. page number and order of valid publication of names in the original articles). Taxonomic opinions included in this List (i.e. the creation of synonyms or the emendation of circumscriptions) cannot be considered as validly published nor, in any other way, approved by the International Committee on Systematics of Prokaryotes and its Judicial Commission.

\begin{tabular}{|c|c|c|}
\hline Name/author(s): & Proposed as: & Page no. \\
\hline 'Candidatus Mycoplasma haematoparvum' Sykes et al. 2005 & Candidatus ${ }^{\star}$ & 29 \\
\hline Lactobacillus suntoryeus Cachat and Priest 2005 & sp. nov. & 33 \\
\hline Lactobacillus rossiae corrig. Corsetti et al. $2005 \dagger$ & sp. nov. & 39 \\
\hline Roseisalinus Labrenz et al. 2005 & gen. nov. & 45 \\
\hline Roseisalinus antarcticus Labrenz et al. 2005 & sp. nov. & 46 \\
\hline Winogradskyella Nedashkovskaya et al. 2005 & gen. nov. & 51 \\
\hline Winogradskyella thalassocola Nedashkovskaya et al. 2005 & sp. nov. & 52 \\
\hline Winogradskyella epiphytica Nedashkovskaya et al. 2005 & sp. nov. & 53 \\
\hline Winogradskyella eximia Nedashkovskaya et al. 2005 & sp. nov. & 54 \\
\hline Microbacterium halotolerans Li et al. 2005 & sp. nov. & 69 \\
\hline Erythrobacter seohaensis Yoon et al. 2005 & sp. nov. & 74 \\
\hline Erythrobacter gaetbuli Yoon et al. 2005 & sp. nov. & 74 \\
\hline Lactobacillus gastricus Roos et al. 2005 & sp. nov. & 80 \\
\hline Lactobacillus antri Roos et al. 2005 & sp. nov. & 80 \\
\hline Lactobacillus kalixensis Roos et al. 2005 & sp. nov. & 81 \\
\hline Lactobacillus ultunensis Roos et al. 2005 & sp. nov. & 81 \\
\hline Lactobacillus satsumensis Endo and Okada 2005 & sp. nov. & 85 \\
\hline Citricoccus alkalitolerans $\mathrm{Li}$ et al. 2005 & sp. nov. & 88 \\
\hline Planococcus stackebrandtii Mayilraj et al. 2005 & sp. nov. & 93 \\
\hline Tenuibacillus Ren and Zhou 2005 & gen. nov. & 98 \\
\hline Tenuibacillus multivorans Ren and Zhou 2005 & sp. nov. & 98 \\
\hline Desulfovibrio putealis Basso et al. 2005 & sp. nov. & 104 \\
\hline Thalassobacter Macián et al. 2005 & gen. nov. & 109 \\
\hline Thalassobacter stenotrophicus Macián et al. 2005 & sp. nov. & 109 \\
\hline Bacillus arenosi Heyrman et al. 2005 & sp. nov. & 114 \\
\hline Bacillus arvi Heyrman et al. 2005 & sp. nov. & 115 \\
\hline Bacillus humi Heyrman et al. 2005 & sp. nov. & 115 \\
\hline Bacillus muralis Heyrman et al. 2005 & sp. nov. & 128 \\
\hline $\begin{array}{l}\text { Bacillus simplex (ex Meyer and Gottheil 1901) Priest et al. } \\
1989 \text { emend. Heyrman et al. } 2005\end{array}$ & emend. $\ddagger$ & 129 \\
\hline Chryseobacterium daecheongense Kim et al. 2005 & sp. nov. & 136 \\
\hline
\end{tabular}


cont.

\begin{tabular}{|c|c|c|}
\hline Name/author(s): & Proposed as: & Page no. \\
\hline Kocuria carniphila Tvrzová et al. 2005 & sp. nov. & 140 \\
\hline Marinobacter bryozoorum Romanenko et al. 2005 & sp. nov. & 145 \\
\hline Marinobacter sediminum Romanenko et al. 2005 & sp. nov. & 147 \\
\hline Halorubrum alkaliphilum Feng et al. 2005 & sp. nov. & 151 \\
\hline Agromyces salentinus Jurado et al. 2005 & sp. nov. & 156 \\
\hline Agromyces neolithicus Jurado et al. 2005 & sp. nov. & 157 \\
\hline Bacillus saliphilus Romano et al. 2005 & sp. nov. & 162 \\
\hline Pontibacillus Lim et al. 2005 & gen. nov. & 168 \\
\hline Pontibacillus chungwhensis Lim et al. 2005 & sp. nov. & 169 \\
\hline Psychrobacter alimentarius Yoon et al. 2005 & sp. nov. & 175 \\
\hline Pibocella Nedashkovskaya et al. 2005 & gen. nov. & 179 \\
\hline Pibocella ponti Nedashkovskaya et al. 2005 & sp. nov. & 179 \\
\hline Lebetimonas Takai et al. 2005 & gen. nov. & 188 \\
\hline Lebetimonas acidiphila Takai et al. 2005 & sp. nov. & 188 \\
\hline Bacillus velezensis Ruiz-García et al. 2005 & sp. nov. & 194 \\
\hline $\begin{array}{l}\text { Pasteurella aerogenes McAllister and Carter } 1974 \text { (Approved Lists 1980) emend. } \\
\text { Christensen et al. } 2005\end{array}$ & emend. $\neq$ & 220 \\
\hline Pasteurella mairii corrig. Sneath and Stevens 1990 emend. Christensen et al. 2005 & emend. $\ddagger$ & 221 \\
\hline Actinobacillus rossii Sneath and Stevens 1990 emend. Christensen et al. 2005 & emend. $\ddagger$ & 221 \\
\hline Aquimarina Nedashkovskaya et al. 2005 & gen. nov. & 227 \\
\hline Aquimarina muelleri Nedashkovskaya et al. 2005 & sp. nov. & 227 \\
\hline Stanierella Nedashkovskaya et al. 2005 & gen. nov. & 227 \\
\hline $\begin{array}{l}\text { Stanierella latercula (Lewin 1969) Nedashkovskaya et al. } 2005 \text { [basonym Cytophaga } \\
\text { latercula Lewin } 1969 \text { (Approved Lists 1980)] }\end{array}$ & comb. nov. & 228 \\
\hline Roseivirga Nedashkovskaya et al. 2005 & gen. nov. & 232 \\
\hline Roseivirga ehrenbergii Nedashkovskaya et al. 2005 & sp. nov. & 233 \\
\hline Salegentibacter mishustinae Nedashkovskaya et al. 2005 & sp. nov. & 237 \\
\hline Salinimonas Jeon et al. 2005 & gen. nov. & 241 \\
\hline Salinimonas chungwhensis Jeon et al. 2005 & sp. nov. & 242 \\
\hline Cryptanaerobacter Juteau et al. 2005 & gen. nov. & 248 \\
\hline Cryptanaerobacter phenolicus Juteau et al. 2005 & sp. nov. & 249 \\
\hline Advenella Coenye et al. 2005 & gen. nov. & 254 \\
\hline Advenella incenata Coenye et al. 2005 & sp. nov. & 255 \\
\hline Streptomyces koyangensis Lee et al. 2005 & sp. nov. & 260 \\
\hline Streptococcus marimammalium Lawson et al. 2005 & sp. nov. & 272 \\
\hline Marinomonas pontica Ivanova et al. 2005 & sp. nov. & 278 \\
\hline Methylobacterium hispanicum Gallego et al. 2005 & sp. nov. & 284 \\
\hline Methylobacterium aquaticum Gallego et al. 2005 & sp. nov. & 285 \\
\hline Spiroplasma atrichopogonis Koerber et al. 2005 & sp. nov. & 291 \\
\hline 'Candidatus Phytoplasma pini' Schneider et al. 2005 & Candidatus $^{*}$ & 306 \\
\hline Marinomonas ushuaiensis Prabagaran et al. 2005 & sp. nov. & 311 \\
\hline Lentibacillus juripiscarius Namwong et al. 2005 & sp. nov. & 319 \\
\hline Gillisia mitskevichiae Nedashkovskaya et al. 2005 & sp. nov. & 322 \\
\hline Methanobacterium beijingense Ma et al. 2005 & sp. nov. & 327 \\
\hline Moraxella oblonga Xie and Yokota 2005 & sp. nov. & 332 \\
\hline Photobacterium lipolyticum Yoon et al. 2005 & sp. nov. & 338 \\
\hline Hydrogenophaga defluvii Kämpfer et al. 2005 & sp. nov. & 343 \\
\hline Hydrogenophaga atypica Kämpfer et al. 2005 & sp. nov. & 343 \\
\hline Marinilactibacillus piezotolerans Toffin et al. 2005 & sp. nov. & 349 \\
\hline Avibacterium Blackall et al. 2005 & gen. nov. & 359 \\
\hline $\begin{array}{l}\text { Avibacterium gallinarum (Hall et al. 1955) Blackall et al. } 2005 \text { [basonym Pasteurella } \\
\text { gallinarum Hall et al. } 1955 \text { (Approved Lists 1980)] }\end{array}$ & comb. nov. & 359 \\
\hline
\end{tabular}


cont.

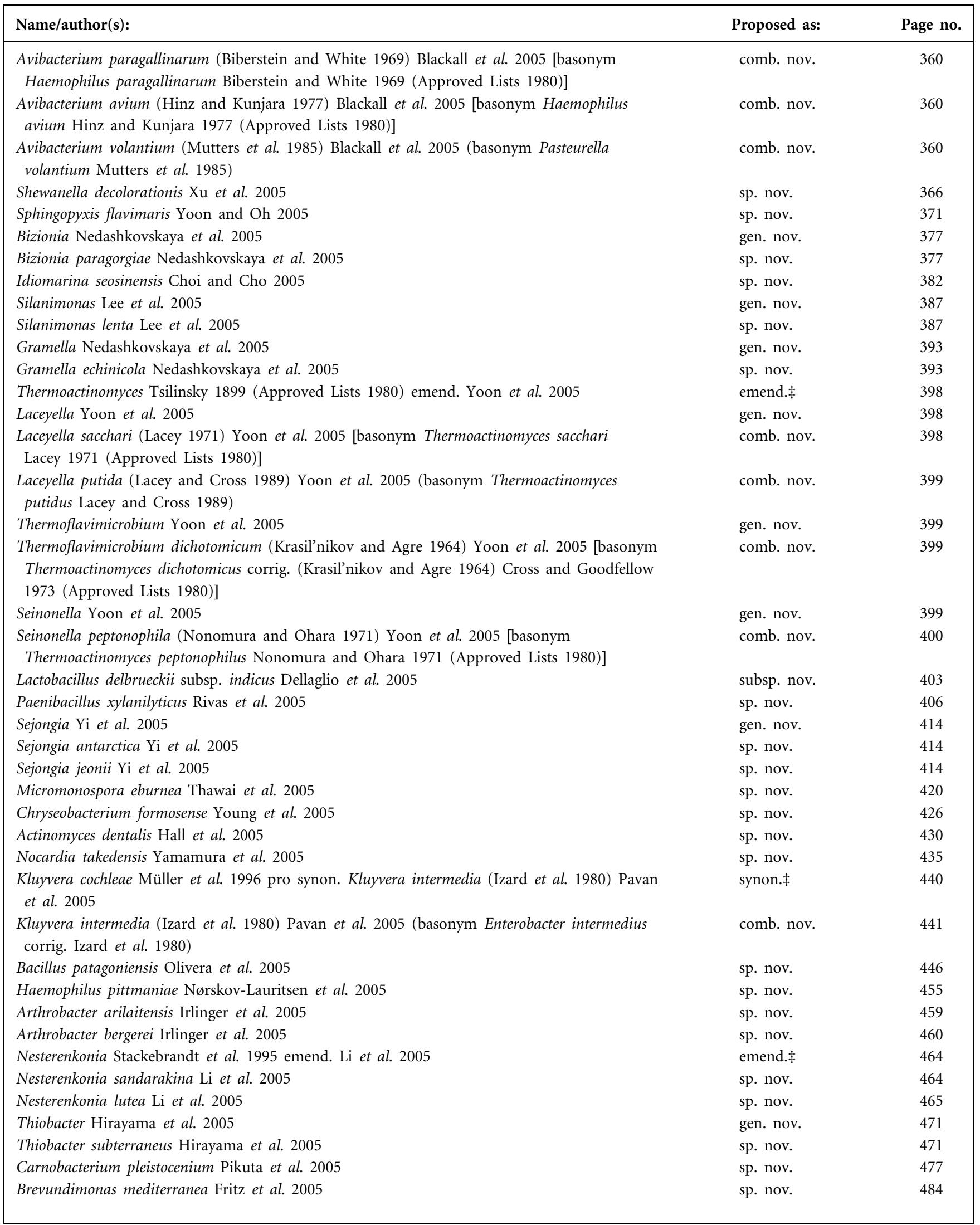


cont.

\begin{tabular}{|c|c|c|}
\hline Name/author(s): & Proposed as: & Page no. \\
\hline Pelczaria Poston 1994 & nom. rej. & 515 \\
\hline Pelczaria aurantia Poston 1994 & nom. rej. & 515 \\
\hline Salmonella enterica (ex Kauffmann and Edwards 1952) Le Minor and Popoff 1987 & sp. nov., nom rev. & 519 \\
\hline $\begin{array}{l}\text { enterica in Salmonella enterica (ex Kauffmann and Edwards 1952) Le Minor and } \\
\text { Popoff } 1987\end{array}$ & nom. cons. & 519 \\
\hline $\begin{array}{l}\text { Salmonella enterica subsp. enterica (ex Kauffmann and Edwards 1952) Le Minor } \\
\text { and Popoff } 1987\end{array}$ & subsp. nov. & 519 \\
\hline $\begin{array}{l}\text { Salmonella enterica subsp. arizonae (Borman 1957) Le Minor and Popoff } 1987 \\
\text { [basonym Salmonella arizonae (Borman 1957) Kauffmann } 1964 \text { (Approved Lists } \\
\text { 1980)] }\end{array}$ & comb. nov. & 519 \\
\hline $\begin{array}{l}\text { Salmonella enterica subsp. bongori (Le Minor et al. 1985) Le Minor and Popoff } 1987 \\
\text { (basonym Salmonella choleraesuis subsp. bongori corrig. Le Minor et al. 1985) }\end{array}$ & comb. nov. & 519 \\
\hline $\begin{array}{l}\text { Salmonella enterica subsp. diarizonae (Le Minor et al. 1985) Le Minor and Popoff } 1987 \\
\text { (basonym Salmonella choleraesuis subsp. diarizonae corrig. Le Minor et al. 1985) }\end{array}$ & comb. nov. & 519 \\
\hline $\begin{array}{l}\text { Salmonella enterica subsp. houtenae (Le Minor et al. 1985) Le Minor and Popoff } 1987 \\
\text { (basonym Salmonella choleraesuis subsp. houtenae corrig. Le Minor et al. 1985) }\end{array}$ & comb. nov. & 519 \\
\hline $\begin{array}{l}\text { Salmonella enterica subsp. indica (Le Minor et al. 1987) Le Minor and Popoff } 1987 \\
\text { (basonym Salmonella choleraesuis subsp. indica Le Minor et al. 1987) }\end{array}$ & comb. nov. & 519 \\
\hline $\begin{array}{l}\text { Salmonella enterica subsp. salamae (Le Minor et al. 1985) Le Minor and Popoff } 1987 \\
\text { (basonym Salmonella choleraesuis subsp. salamae corrig. Le Minor et al. 1985) }\end{array}$ & comb. nov. & 519 \\
\hline $\begin{array}{l}\text { Salmonella choleraesuis corrig. (Smith 1894) Weldin } 1927 \text { (Approved Lists 1980) synon. } \\
\text { Salmonella enterica (ex Kauffmann and Edwards 1952) Le Minor and Popoff } 1987\end{array}$ & synon. & 523 \\
\hline $\begin{array}{l}\text { Salmonella enteritidis (Gaertner 1888) Castellani and Chalmers } 1919 \text { (Approved Lists } \\
\text { 1980) synon. Salmonella enterica subsp. enterica (ex Kauffmann and Edwards 1952) } \\
\text { Le Minor and Popoff } 1987\end{array}$ & synon. $\ddagger$ & 523 \\
\hline $\begin{array}{l}\text { Salmonella paratyphi (ex Kayser 1902) Ezaki et al. 2000. synon. Salmonella enterica subsp. } \\
\text { enterica (ex Kauffmann and Edwards 1952) Le Minor and Popoff } 1987\end{array}$ & synon. $\ddagger$ & 523 \\
\hline $\begin{array}{l}\text { Salmonella typhi (Schroeter 1886) Warren and Scott } 1930 \text { (Approved Lists 1980) synon. } \\
\text { Salmonella enterica subsp. enterica (ex Kauffmann and Edwards 1952) Le Minor and } \\
\text { Popoff } 1987\end{array}$ & synon. $\ddagger$ & 523 \\
\hline $\begin{array}{l}\text { Salmonella typhimurium (Loeffler 1892) Castellani and Chalmers } 1919 \text { (Approved } \\
\text { Lists 1980) synon. Salmonella enterica subsp. enterica (ex Kauffmann and Edwards } \\
\text { 1952) Le Minor and Popoff } 1987\end{array}$ & synon. $\neq$ & 523 \\
\hline $\begin{array}{l}\text { Salmonella choleraesuis subsp. choleraesuis corrig. (Smith 1894) Weldin } 1927 \text { synon. } \\
\text { Salmonella enterica subsp. enterica (ex Kauffmann and Edwards 1952) Le Minor } \\
\text { and Popoff } 1987\end{array}$ & synon. $\neq$ & 524 \\
\hline Thioalkalicoccus Bryantseva et al. 2000 & Deleted correction $\$$ & 528 \\
\hline Thioalkalicoccus limnaeus Bryantseva et al. 2000 & Deleted correction (generic name) $\S$ & 528 \\
\hline Thioalkalimicrobium Sorokin et al. 2001 & Deleted correction $\$$ & 528 \\
\hline Thioalkalimicrobium aerophilum Sorokin et al. 2001 & Deleted correction (generic name) $\$$ & 528 \\
\hline Thioalkalimicrobium cyclicum Sorokin et al. 2002 & Deleted correction (generic name) $\S$ & 528 \\
\hline Thioalkalimicrobium sibiricum Sorokin et al. 2001 corrig. & Deleted correction (generic name)॥ & 528 \\
\hline Thioalkalivibrio Sorokin et al. 2001 & Deleted correction $\S$ & 528 \\
\hline Thioalkalivibrio denitrificans Sorokin et al. 2001 & Deleted correction (generic name) $\$$ & 528 \\
\hline Thioalkalivibrio jannaschii Sorokin et al. 2002 & Deleted correction (generic name) $\S$ & 528 \\
\hline Thialkalivibrio nitratireducens Sorokin et al. 2003 & corrig. 9 & 528 \\
\hline Thioalkalivibrio nitratis Sorokin et al. 2001 & Deleted correction (generic name) $\S$ & 528 \\
\hline Thioalkalivibrio paradoxus Sorokin et al. 2002 & Deleted correction (generic name) $\S$ & 528 \\
\hline Thioalkalivibrio thiocyanoxidans Sorokin et al. 2002 & Deleted correction (generic name) $\S$ & 528 \\
\hline Thioalkalivibrio versutus Sorokin et al. 2001 & Deleted correction (generic name) $\S$ & 528 \\
\hline
\end{tabular}


${ }^{\star}$ Category not covered by the Rules of the Bacteriological Code (1990 Revision).

$\dagger$ The original spelling of the specific epithet, rossii (sic), has been corrected on notification with the authors' agreement.

$\ddagger$ Taxonomic opinion.

\$The names Thioalkalicoccus, Thioalkalicoccus limnaeus, Thioalkalimicrobium, Thioalkalimicrobium aerophilum, Thioalkalimicrobium cyclicum, Thioalkalivibrio, Thioalkalivibrio denitrificans, Thioalkalivibrio jannaschii, Thioalkalivibrio nitratis, Thioalkalivibrio paradoxus, Thioalkalivibrio thiocyanoxidans and Thioalkalivibrio versutus were corrected to Thialkalicoccus, Thialkalicoccus limnaeus, Thialkalimicrobium, Thialkalimicrobium aerophilum, Thialkalimicrobium cyclicum, Thialkalivibrio, Thialkalivibrio denitrificans, Thialkalivibrio jannaschii, Thialkalivibrio nitratis, Thialkalivibrio paradoxus, Thialkalivibrio thiocyanoxidans and Thialkalivibrio versutus, respectively [Int J Syst Evol Microbiol 51 (2001), 267-268; Int J Syst Evol Microbiol 51 (2001), 795-796; Int J Syst Evol Microbiol 52 (2002), 691-692; Int J Syst Evol Microbiol 52 (2002), 1077-1079]. During the meeting of the Judicial Commission held during the Xth International (IUMS) Congress of Bacteriology and Applied Microbiology, H. G. Trüper proposed that the recommendations governing the use of the connecting vowel should be overruled by the usage in chemistry and physics. Consequently, in combined names that include terms used in chemistry or physics the connecting vowel shall not be dropped when the following component starts with a vowel and when this may cause ambiguity or uncertainty in meaning. The Judicial Commission unanimously agreed to this proposal and the original spellings Thioalkalicoccus, Thioalkalimicrobium, and Thioalkalivibrio must be used.

IIThioalkalimicrobium sibericum was corrected to Thialkalimicrobium sibiricum [Int J Syst Evol Microbiol 51 (2001), 795-796]. According to the Judicial Commission (see footnote $\$$ ), the original spelling of the generic name must be used. However, the correction of the specific epithet is maintained, and the name should be cited as Thioalkalimicrobium sibiricum corrig. Sorokin et al. 2001.

In 2003, Sorokin et al. validly published the name Thialkalivibrio nitratireducens. According to the Judicial Commission (see footnote $\$$ ), Thialkalivibrio nitratireducens must be corrected to Thioalkalivibrio nitratireducens. 